\title{
Исследование электрофизических свойств квантовых точек антимонида индия: значение формы
}

\author{
(C) В.Ф. Кабанов, А.И. Михайлов, М.В. Гавриков Ф \\ Саратовский государственный университет им. Н.Г. Чернышевского, \\ 410012 Саратов, Россия \\ ฯ E-mail: maks.gavrikov.96@gmail.com
}

Поступила в Редакцию 22 июня 2020 г.

В окончательной редакции 5 октября 2020 г.

Принята к публикации 5 октября 2020 г.

\begin{abstract}
Проведено исследование влияния формы квантовых точек антимонида индия на некоторые важные электрофизические параметры методами анализа спектральных характеристик, просвечивающей электронной микроскопии, сканирующей туннельной микроскопии, с применением лазерного анализатора размера частиц, методами сканирующей электронной микроскопии. Показано, что реальная форма квантовых точек (шаровая и кубическая модели) при одном и том же характерном размере будет заметно влиять на энергетический спектр рассматриваемых объектов и соответственно на их электрофизические и оптические свойства.
\end{abstract}

Ключевые слова: антимонид индия, квантовые точки, шаровая и кубическая модели, энергетический спектр электронов.

DOI: 10.21883/FTP.2021.03.50601.9471

\section{1. Введение}

Электронные и оптические свойства полупроводниковых квантовых точек (КТ) и структур, включающих их, существенно определяются материалом, характерным размером полупроводниковых наночастиц и их формой. С практической точки зрения важными являются следующие оптические диапазоны: видимый, длины волн 400-750 нм; ближний инфракрасный, 800-900 нм, окно прозрачности крови; 1300-1550 нм, телекоммуникационный диапазон.

Квантовые точки на основе соединений $\mathrm{A}^{\mathrm{III}} \mathrm{B}^{\mathrm{V}}$ и $\mathrm{A}^{\mathrm{II}} \mathrm{B}^{\mathrm{VI}}$ имеют большую силу осциллятора оптических переходов, что приводит к коротким (субнаносекундным) временам рекомбинации электронно-дырочных пар [1]. Также следует отметить температурную стабильность излучения, наблюдаемого до комнатных температур. Квантовые точки на основе антимонида индия (InSb) вызывают особый интерес благодаря уникальным свойствам материала: прежде всего - это узкая прямая запрещенная зона $(0.17$ эВ), малые эффективные массы электронов и дырок (для электронов $0.013 m_{0}$, $m_{0}$ - масса свободного электрона [2-4]), большая длина волны де Бройля электрона, до $\sim 55$ нм. В этом случае в диапазоне изменяемых размеров КТ от 30 до 5 нм величина энергетического зазора $\varepsilon_{c_{1}}-\varepsilon_{v_{1}}$ с учетом ширины запрещенной зоны объемного материала будет изменяться в диапазоне от 0.3 до 2.8 эВ (или положение максимума спектральных характеристик от 500 до 4000 нм). Это позволяет обоснованно предполагать возможность значительного влияния на оптические и электрические свойства KT InSb в широком диапазоне при управляемом изменении характерных размеров КТ.

Определенное влияние на свойства квантовых точек, помимо состава и размера, будет оказывать их фор- ма [5]. Например, при одинаковых характерных размерах КТ антимонида индия (диаметр шара или ребро куба $\sim(10-15)$ нм), положение первого энергетического уровня $\varepsilon_{c_{1}}$, отсчитываемое от уровня энергии дна зоны проводимости объемного материала, для сферической и для кубической моделей в приближении трехмерной бесконечно глубокой потенциальной ямы может теоретически различаться на сотни мэВ.

Цель настоящей работы - исследование влияния формы КТ на электрофизические и оптические свойства пленочной структуры коллоидных квантовых точек антимонида индия.

\section{2. Технологии получения образцов и методика измерений}

Коллоидный синтез ядер КT InSb проводился в среде безводного олеиламина, с использованием в качестве прекурсоров хлорида индия, $\mathrm{InCl}_{3}$, и трис[бис(триметилсилил)амид] сурьмы, $\mathrm{Sb}[\mathrm{N}(\mathrm{Si}-(\mathrm{Me}) 3) 2] 3$, согласно методике [6].

Квантовые точки наносили на стеклянные подложки с проводящим слоем оксида индия-олова (ITO) из коллоидного раствора различными методами при последующем контролируемом испарении растворителя и контроле параметров слоя оптическими методами. Полученные образцы были исследованы методами анализа спектральных характеристик, просвечивающей электронной микроскопии (ПЭМ), сканирующей туннельной микроскопии (СТМ), с применением лазерного анализатора размера частиц, методами сканирующей электронной микроскопии (СЭМ), с использованием автоэмиссионного сканирующего электронного микроскопа MIRA 2 LMU. 
Для дополнительного анализа использовался метод нормированных дифференциальных вольт-амперных характеристик $I(V)$, именно зависимостей $(d I / d V) /(I / V)$ от напряжения $V$, подробно описанный и обоснованный B [7].

В том случае, если энергию электрона в КТ можно представить в виде трехмерной бесконечно глубокой потенциальной ямы и использовать модель КТ кубической формы с ребром $a$, положение уровней энергетического спектра может быть представлено как [8]

$$
\varepsilon_{i}=\frac{(\pi \hbar)^{2}}{2 m^{*}} \frac{1}{a^{2}}\left(i_{x}^{2}+i_{y}^{2}+i_{z}^{2}\right),
$$

где $i_{x}, i_{y}, i_{z}=1,2,3 \ldots ; m^{*}$ - эффективная масса электрона, $a$ - характерный размер КТ (ребро куба).

В случае модели КТ шаровой формы с радиусом $r$ в приближении одноэлектронного спектра в параболической яме положение уровней энергетического спектра может быть представлено как [9]

$$
\varepsilon_{i}=\frac{(\pi \hbar)^{2}}{2 m^{*}} \frac{1}{r^{2}}(4 n+2 l+3),
$$

где $n, l=0,1,2, \ldots$

\section{3. Результаты измерений и их обсуждение}

Типичный экспериментальный спектр поглощения зависимость коэффициента поглощения $\alpha$ от длины волны $\lambda$ - для исследуемых КТ представлена на рис. 1.

В работе проводилось сопоставление размеров частиц, полученных при анализе спектра поглощения КТ

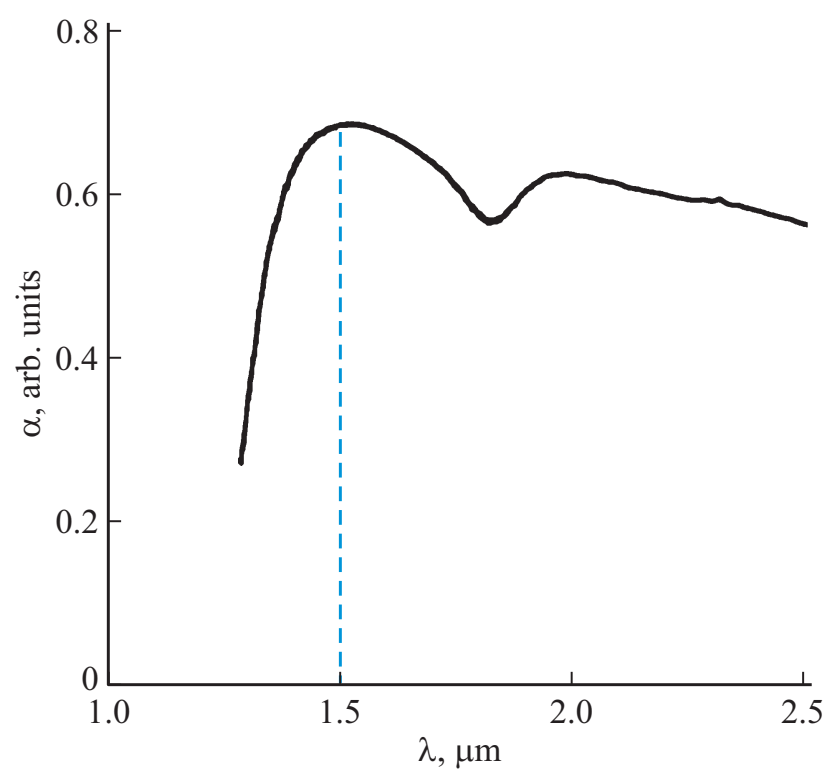

Рис. 1. Типичная экспериментальная зависимость коэффициента поглощения $\alpha$ от длины волны $\lambda$ для исследуемых КТ $\mathrm{InSb}$.

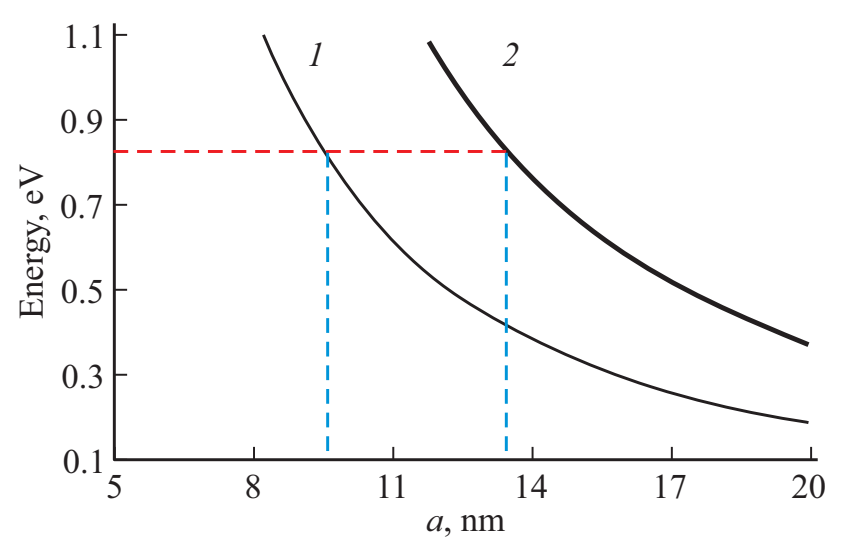

Рис. 2. Расчетные кривые первого энергетического уровня в KT InSb в зависимости от размера КТ для кубической $(1)$ и шаровой (2) моделей.

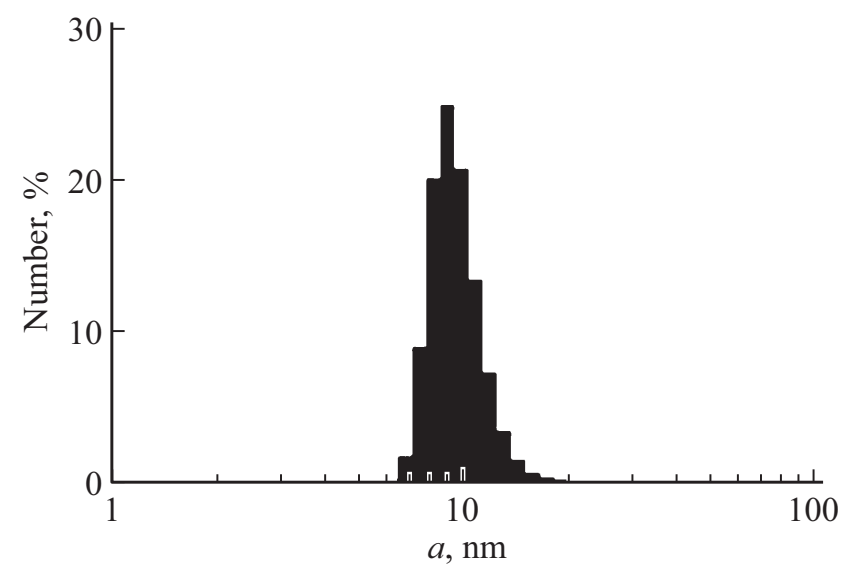

Рис. 3. Результат гранулометрического анализа состава раствора KT InSb в виде функции распределения дисперсной фазы по размерам частиц.

$\mathrm{InSb}$ с использованием различных предельных моделей: куб и шар. Оценка размера частицы по максимуму в спектре поглощения проводилась из следующих соображений. В первом приближении энергия кванта поглощенного излучения в квантовой точке может быть представлена в виде

$$
\varepsilon_{h v}=\varepsilon_{g_{0}}+\varepsilon_{c_{1}}+\varepsilon_{v_{1}},
$$

где $\varepsilon_{h v}-$ энергия максимума поглощения, $\varepsilon_{g_{0}}-$ ширина запрещенной зоны объемного материала, $\varepsilon_{c_{1}}$ энергия первого уровня электронов в квантовой точке, отсчитываемая от дна зоны проводимости объемного материала, $\varepsilon_{v_{1}}$ - энергия первого уровня дырки, отсчитываемая от потолка валентной зоны объемного материала. Энергия максимума с погрешностью не более $2 k T$ $(\sim 0.05$ эВ при комнатных температурах $)$ сопоставлялась с зависимостью $\varepsilon_{c_{1}}$ от характерного размера квантовой точки $a$ (в случае кубической модели $a-$ ребро куба, в случае шаровой модели $a$ - это диаметр шара, $a=2 r$ ). По группе измерений проводилось усреднение. 


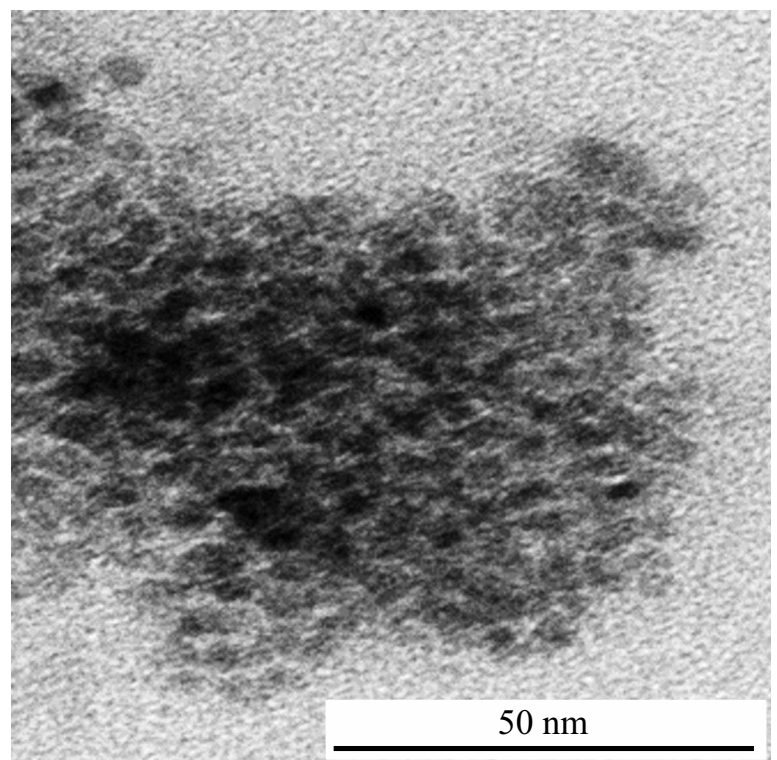

Рис. 4. Типичное ПЭМ-изображение КT InSb.

С учетом того что эффективная масса электрона в рассматриваемом материале $\left(\sim 0.013 m_{0}, m_{0}\right.$ - масса свободного электрона) существенно меньше, чем эффективная масса дырки $\left(\sim 0.42 m_{0}\right)$, в выражении (1) можно пренебречь $\varepsilon_{v_{1}}$ по сравнению с $\varepsilon_{c_{1}}$.

Используя для объемного материала InSb значение $\varepsilon_{g}=0.17$ эВ, положения максимумов на спектральной зависимости для групп КТ $(\lambda \approx 1.5$ мкм соответствует $\varepsilon_{h v} \approx 0.83$ эВ) и формулы (1), (2) для оценки энергии $\varepsilon_{c_{1}}$, можно провести оценку характерных размеров квантовых точек. Рассчитанные значения энергии электрона для первого уровня КT InSb по кубической и шаровой моделям представлены на рис. 2. Горизонтальной прямой на рисунке показано положение экспериментально определенного максимума поглощения (в эВ), вертикальными линиями - соответствующие данному максимуму размеры КТ. Сравнение экспериментально определенного положения максимума поглощения с теоретическими кривыми показало значение в диапазоне 9-10 в нм для кубической модели и 13-14 нм для шаровой модели.

Результат независимого определения характерных размеров исследованных КТ с помощью лазерного анализатора приведен на рис. 3. Измерения показали размер наночастиц в диапазоне $8-10$ нм, что с большой долей вероятности соответствует кубической модели КТ (рис. 2).

С целью подтверждения или опровержения этого вывода пленочные образцы были исследованы методами просвечивающей электронной микроскопии (ПЭМ). Типичное ПЭМ-изображение КT InSb представлено на рис. 4. Анализ формы KT InSb позволил сделать вывод, что исследуемые объекты можно отнести к классу нанокристаллов со сложной геометрией, преимущественно кубической формы.

Реальная форма КТ при определенном размере будет заметно влиять на электронный спектр рассматриваемых объектов и на электрофизические и оптические свойства. Это можно показать на примере, представленном на рис. 5, где приведено соответствие 1-го и 2-го пиков на нормированной дифференциальной туннельной ВАХ рассчитанным зависимостям энергий первого и второго уровней электронов в КТ для кубической модели. Данные пики характерны для туннелирования электронов через уровни КТ в металлический зонд туннельного микроскопа [10-12]. Характерный размер КТ, определенный таким образом, составляет 8-10 нм. Как видно, эти данные также хорошо коррелируют с приведенными выше рассуждениями.

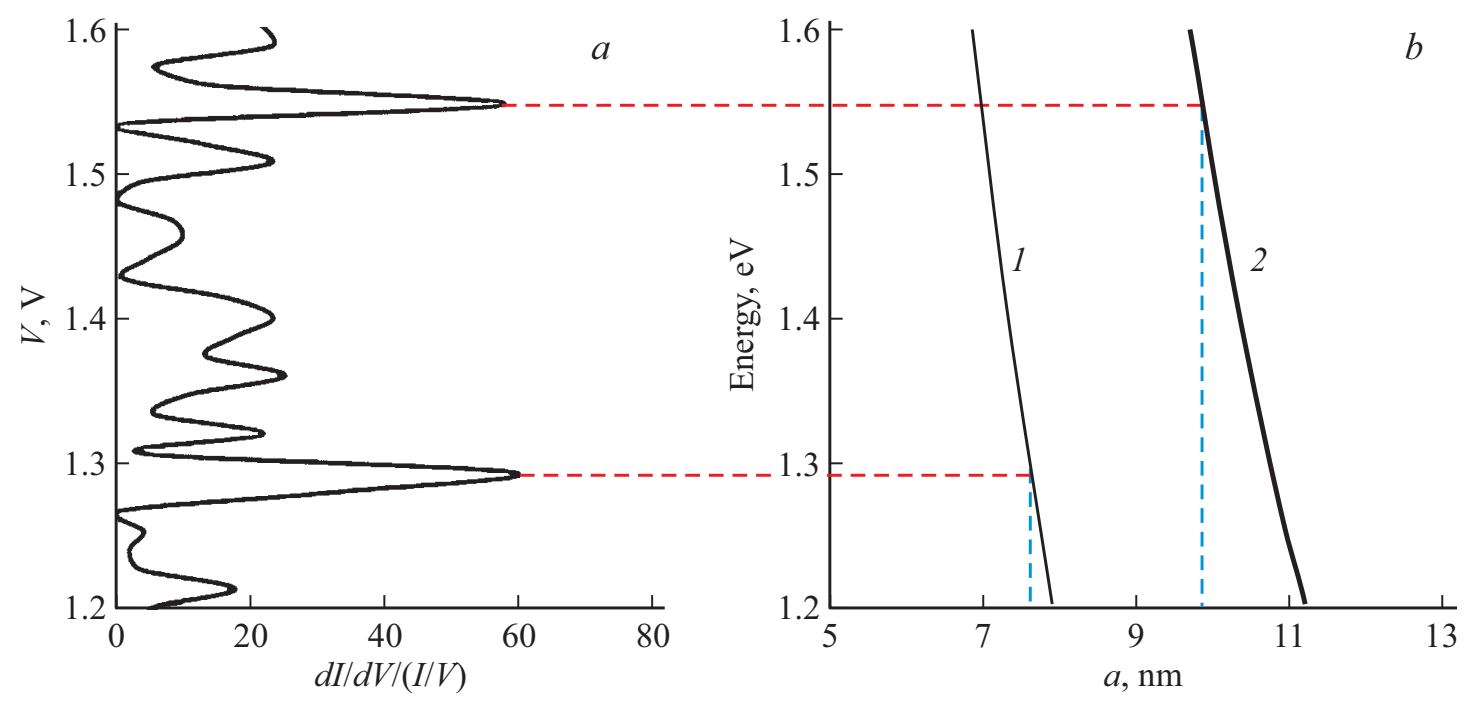

Рис. 5. Типичная дифференциальная туннельная $\mathrm{BAX} \mathrm{KT} \operatorname{InSb}(a)$, энергии первого (1) и второго (2) уровней электронного спектра KT InSb в зависимости от размера, рассчитанные по кубической модели $(b)$. 


\section{4. Заключение}

Таким образом, в работе проведено исследование влияния формы квантовых точек антимонида индия на некоторые важные электрофизические параметры методами анализа спектральных характеристик, просвечивающей электронной микроскопии, сканирующей туннельной микроскопии, с использованием лазерного анализатора размера частиц, методами сканирующей электронной микроскопии, с использованием автоэмиссионного сканирующего электронного микроскопа MIRA 2 LMU.

Показано, что реальная форма КТ (шаровая и кубическая модели в предельном случае) при одном и том же характерном размере будет заметно влиять на электронный спектр рассматриваемых объектов и соответственно на электрофизические и оптические свойства, что наряду с материалом и размером может рассматриваться как эффективный фактор управления.

\section{Финансирование работы}

Работа выполнена при финансовой поддержке грантов РФФИ № 19-07-00087 и № 19-07-00086.

\section{Конфликт интересов}

Авторы заявляют, что у них нет конфликта интересов.

\section{Список литературы}

[1] И.В. Игнатьев, И.Э. Козин. Динамика носителей в полупроводниковых квантовых точках (СПб., Соло, 2005).

[2] P. Reiss, M. Carriere. Chem. Rev., 116 (18), 10731 (2016).

[3] A.D. Yoffe. Adv. Phys., 50 (1), 1 (2010).

[4] S.B. Brichkin, V.F. Razumov. Russ. Chem. Rev., 85 (12), 1297 (2016).

[5] Р.Б. Васильев, Д.Н. Дирин. Квантовые точки: синтез, свойства, применение (М., ФНМ, 2007).

[6] W. Liu, A.Y. Chang, R.D. Schaller, D.V. Talapin. J. Am. Chem. Soc., 134 (50), 20258 (2012).

[7] A.I. Mikhailov, V.F. Kabanov, E.G. Glukhovskoy, M.I. Shishkin, M.V. Gavrikov. Nanosystems: Phys., Chem., Math., 9, 464 (2018).

[8] В.П. Драгунов, И.Г. Неизвестный, В.А. Гридчин. Основы наноэлектроники (М., Логос, 2006).

[9] N.E. Kaputkina, Y.E. Lozovik. Phys. Solid State, 40 (11), 1935 (1998).

[10] A.I. Mikhailov, V.F. Kabanov, N.D. Zhukov, E.G. Glukhovskoy. Nanosystems: Phys., Chem., Math., 8 (5), 596 (2017).

[11] T. Wang. ACS Nano, 9 (1), 725 (2015).

[12] А.И. Михайлов, В.Ф. Кабанов, И.А. Горбачев, Е.Г. Глуховской. ФТП, 52 (6), 603 (2018).

Редактор Л.В. Шаронова

\section{Study of electrophysical properties of indium antimonide quantum dots: importance of the form}

\author{
V.F. Kabanov, A.I. Mikhailov, M.V. Gavrikov
}

Saratov State University, 410012 Saratov, Russia

Abstract In this work, we studied the influence of the geometrical form of indium antimonide quantum dots on some important electrophysical parameters by means of spectral characteristics analysis, transmission electron microscopy, scanning tunneling microscopy, using a laser particle size analyzer, and by scanning electron microscopy. It is shown that the real form of quantum dots (spherical and cubic models) at the same characteristic size affects noticeably the energy spectrum of the investigated objects and, accordingly, their electrophysical and optical properties. 